\section{MedienPädagogik}

www. medienpaed.com
Zeitschrift für

Theorie und Praxis

der Medienbildung

ISSN 1424-3636

Themenheft Nr. 14: Qualitative Forschung in der Medienpädagogik

\title{
Rekonstruktionslogische Forschung für die Mediendidaktik
}

Kerstin Mayrberger

Dieser Beitrag setzt sich mit der rekonstruktiven bzw. rekonstruktionslogischen Perspektive qualitativer Forschung und deren Bedeutung für die Mediendidaktik als einem zentralen Teilgebiet der Medienpädagogik auseinander. Im Zentrum des Beitrags steht die Auseinandersetzung mit der Methode und Methodologie der objektiven Hermeneutik nach Oevermann und deren Potenzial und Grenzen für eine am qualitativen Forschungsparadigma orientierte mediendidaktische Forschung. Exemplarischer Bezugsrahmen stellt ein Forschungsprojekt dar, das die gemeinschaftlichen Lernprozesse von Grundschülern/-innen beim Arbeiten mit dem Computer in einer Medienecke im alltäglichen, geöffneten Unterricht untersucht.

\section{Einleitung}

Ein wesentliches Kennzeichen der Medienpädagogik ist ihre Interdisziplinarität (vgl. u. a. Hüther/Schorb 2005). In Folge dessen lässt sich auch die medienpädagogische Forschung zwischen den Disziplinen verorten. Der vorliegende Beitrag knüpft hier an, indem die Frage nach dem Potenzial einer empirisch rekonstruktiven bzw. rekonstruktionslogischen Herangehensweise an mediendidaktische Fragestellungen mit Bezug auf die qualitative (Grund-)Schul- bzw. Unterrichtsforschung erörtert wird. Kern des vorliegenden Beitrags ist die theoretische Auseinandersetzung mit dieser Fragestellung unter Rückgriff auf die objektive Hermeneutik (vgl. u. a. Oevermann 1979, 2002). Dafür wird exemplarisch Bezug genommen auf ein Forschungsprojekt, das die gemeinschaftlichen Lernprozesse von Grundschülerinnen und Grundschülern beim Arbeiten mit dem Computer in einer Medienecke im alltäglichen, geöffneten Unterricht untersucht.

\section{Die mediendidaktische Perspektive in der Medienpädagogik}

Die Medienpädagogik wird auch heute noch in Anlehnung an die «klassische Zweiteilung» nach Kösel/Brunner grob in Mediendidaktik (Pädagogik mit Medien) und Medienerziehung bzw. Medienpädagogik im engeren Sinne (Pädagogik über Medien) differenziert (Hüther 2005, S. 235). Im Kontext von Lehren und Lernen lässt sich vor allem mit Einzug der neuen bzw. digitalen Medien in die (medien-)pädagogischen Praxis die grobe Unterscheidung von Medienerziehung und Mediendidaktik so nicht mehr klar aufrecht halten (vgl. u.a. Tulodziecki/Herzig 2004; Hüther 2005). Aus theoretischer Perspektive erscheint es dennoch sinnvoll zu differenzieren, weshalb im Folgenden auf Perspektiven zum Lehren und Lernen mit 
Unterstützung von digitalen Medien verwiesen wird, die in ihrer Zusammenschau wesentliche Aspekte der Mediendidaktik aufzeigen:

Sacher (2000, S. 15) charakterisiert den Teil der Medienpädagogik, der sich mit der didaktischen Medienverwendung und -gestaltung beschäftigt wie folgt: «Sie zielt auf die Unterstützung nicht medienthematischer Lehr- und Lernprozesse durch Medien, d. h. solcher Lehr- und Lernprozesse, in denen Medien lediglich Vehikel bzw. Mittel, nicht aber selbst Gegenstand des Lehrens und Lernens sind.» Nach Hüther (2005, S. 237) befasst sich Mediendidaktik «mit Fragen der Mediengestaltung und -auswahl. Sie trifft Aussagen über die didaktischen Funktionen, die Medien in LehrLernsituationen übernehmen können und sie untersucht die unterschiedlichen Formen, in denen Bildungsmedien zum Einsatz kommen. Sie will letztlich die Basis für didaktisch begründete Medienentscheidungen liefern.» Dahinter steht die Annahme, dass Medienentscheidungen primär didaktische Entscheidungen sein sollten, «bei denen der Medieneinsatz den unterrichtlichen Notwendigkeiten folgt und nicht der Unterricht den medialen Gegebenheiten und Möglichkeiten» (ebd.). Allgemeiner beschreiben schliesslich Tulodziecki/Herzig (2004, S. 249) Mediendidaktik als den Bereich der Didaktik, «in dem alle Überlegungen zusammengefasst sind, bei denen es im Wesentlichen um die Frage geht, wie Medien bzw. Medienangebote oder Medienbeiträge zur Erreichung pädagogisch gerechtfertigter Ziele gestaltet und verwendet werden können bzw. sollen».

Diese Begriffsbestimmungen verdeutlichen in erster Linie, dass die Entscheidung über die Gestaltung, Auswahl, Verwendung und Integration von Medien in Lehr- und Lernprozesse durch (medien-)pädagogisch Handelnde (medien-)didaktisch wohl begründet erfolgen sollten. So sei hier exemplarisch auf das Lernumgebungskonzept (vgl. ebd., S. $112 \mathrm{ff}$.) als ein zeitgemässes mediendidaktisches Konzept verwiesen, in dem traditionelle wie moderne Medien wichtige Elemente darstellen. Eine solche Lernumgebung zeichnet sich u. a. dadurch aus, dass sie in der Regel von Lehrenden so gestaltet wird, dass diese die Lernenden in ihrem Lernprozess darin unterstützt, sich aktiv mit bedeutsamen Aufgaben auseinanderzusetzen (vgl. dazu auch Dörr/Strittmatter 2002; Kron/Sofos 2003). Der Frage nach der (sinnvollen) Unterstützung von Lernprozessen in einer (computerunterstützten) Lernumgebung kann nun aus einer rekonstruktionslogischen Perspektive begegnet werden, wie die folgende (Kurz-)Darstellung einer empirischen Studie zeigt.

\section{Exemplarischer Bezugsrahmen: Empirische Untersuchung zum veränderten Lernen mit neuen Medien}

Mit dem Einzug der neuen bzw. digitalen Medien in Form von Computer und Internet in den Unterricht erweitern sich das Spektrum der didaktischen Möglichkeiten von Lernumgebungen und damit einhergehend auch die Ansprüche an deren Gestaltung. Es werden in diesem Zuge begründete Forderungen nach einem «neuen» oder «veränderten Lernen» «mit neuen Medien» laut, die sich in einer 
veränderten Lehr- und Lernkultur ausdrücken (sollen) (vgl. u. a. Aufenanger 1999, Reinmann-Rothmeier/Mandl 2001; Schwetz/Zeyringer/Reiter 2001; Schulz-Zander 2005; Mitzlaff 2007). Interessant ist in diesem Zusammenhang die Frage, wie entsprechende Lernprozesse im Rahmen von alltäglichen, computerunterstützten Unterricht gestaltet und gefördert werden können.

In diesem Sinne wurden im Rahmen eines Forschungsprojekts (vgl. Mayrberger 2007) gemeinschaftliche Lernprozesse von Schülern/-innen beim Arbeiten mit dem Computer in der Medienecke im alltäglichen, geöffneten Unterricht der Grundschule aus einer (mikro-)soziologischen Perspektive untersucht. Die empirische Studie hatte zum Ziel, die konstitutiven Strukturen sozialen Handelns während der gemeinschaftlichen Interaktionsprozesse in einer computerunterstützten Lernumgebung zu rekonstruieren. Dabei waren besonders solche beobachtbaren Lernhandlungen von Interesse, die Rückschlüsse auf Interaktionen im Sinne von «Ko-Konstruktion» (vgl. u. a. Reusser 2001) erlauben.

Das Erkenntnisinteresse der Untersuchung bestand aus einer allgemein theoretischen bzw. fachwissenschaftlichen Sicht darin, zu untersuchen, welche Chancen und Schwierigkeiten die Integration der neuen Medien in den schulischen Unterricht für gemeinschaftliche Lernprozesse mit sich bringt. Der weitgehend etablierten Auffassung folgend, dass die Integration der neuen Medien in eine Lernumgebung noch kein verändertes oder «neues» Lernen mit sich bringt, wurde diese Art von Lernumgebung offen und konstruktiv in den Blick genommen. Ziel war es, empirisch begründete Aussagen über die Konstitution einer computerunterstützten, an gemässigt konstruktivistischen Ansätzen orientierten Lernumgebung tätigen zu können und entsprechende Konsequenzen für eine (medien)pädagogisch bzw. mediendidaktisch sinnvolle Gestaltung aufzuzeigen. So wurde allgemein danach gefragt, inwieweit ein neues Lernen mit neuen Medien in einer an gemässigt konstruktivistischen Grundsätzen orientierten Lernumgebung stattfindet bzw. -finden kann. Dieser Frage wurde konkret im Rahmen geöffneten Unterrichts in der Grundschule nachgegangen, da dieser Ansatz eine theoretische Passung zu einer gemässigt konstruktivistischen Auffassung vom Lehren und Lernen (vgl. u. a. Reinmann-Rothmeier/Mandl 2001) aufweist. Das besondere Interesse lag darin, herauszuarbeiten, inwieweit sich ein gemeinschaftliches Lernen im Rahmen einer im Alltag der Grundschule bestmöglichen Lernumgebung entfalten kann. Das «kooperative bzw. kollaborative Lernen» gilt als ein zentrales Merkmal von sich an konstruktivistisch geprägten Ansätzen orientierenden Lernprozessen und wurde hier exemplarisch für ein «neues Lernen» in den Blick genommen. Dem folgend wurde Lernen aus einer vornehmlich soziologischen Perspektive fokussiert, indem Lernen als beobachtbarer sozialer Prozess bzw. Lernen als soziale Handlung betrachtet wurde. Insofern stellte die Kommunikation beim Lernen den zentralen Untersuchungsgegenstand dar. So lautete die zentrale Fragestellung der konkreten empirischen Untersuchung auch, welche Strukturmerkmale (Regeln) 
den Kommunikationsprozessen während des gemeinschaftlichen Lernens in einer computerunterstützten, gemässigt konstruktivistisch orientierten Lernumgebung zu Grunde lägen. Es wurden Aussagen dazu erwartet, in welchem Masse die Chancen, die eine gemässigt konstruktivistische, computerunterstützte Lernumgebung potenziell bietet, auch in gemeinschaftlichen Lernprozessen von den Schülerinnen und Schülern genutzt werden.

Im Folgenden wird nun vor dem Hintergrund der leitenden Fragestellungen theoretisch auf die rekonstruktionslogische Forschung im Kontext von Mediendidaktik eingegangen, bevor die konkrete Studie mit ihrem Forschungsdesign weiter ausgeführt und zur weiteren Erörterung herangezogen wird.

\section{Rekonstruktionslogische Forschung und Mediendidaktik}

Ansätze qualitativer Sozialforschung orientieren sich u. a. an einer rekonstruktiven Methodologie (vgl. Flick et al. 2000, S. 20f.). So liegt rekonstruktiven Ansätzen die Annahme zu Grunde, dass Wirklichkeit ein gesellschaftliches Konstrukt darstellt. Diese Konstruktion von Wirklichkeit, die in den Handlungen der Akteure nach impliziten Regeln sozialen Handelns vollzogen wird, gilt es zu rekonstruieren. Forschungspraktisch bezieht sich ein rekonstruktives Vorgehen auf Beobachtungen von Handlungen oder symbolischen Repräsentationen, wie z. B. verbalen oder visuellen Daten (vgl. u. a. Oevermann 2002, Bohnsack 2003). So wird hier eine Forschungsperspektive eingenommen, die sich mit der Rekonstruktion handlungsgenerierender Strukturen bzw. der hermeneutischen Analyse tiefer liegender Strukturen beschäftigt. Entsprechend geht es in solchen Untersuchungen nicht um den Nachvollzug des subjektiv gemeinten Sinns (Symbolischer Interaktionismus) oder die Deskription von Prozessen der Herstellung sozialer Situationen und Milieus (Ethnomethodologie) (vgl. Flick 1999, S. 28 ff.; Flick et. al 2000, S. 20 f.). Allgemein zeichnet sich ein rekonstruktionslogisches Vorgehen im Gegensatz zur Interpretation bzw. Deutung oder Intervention dadurch aus, dass hier «der subjektive Sinnhorizont überschritten wird in Richtung auf Sinnsphären, die von den Handelnden mental nicht repräsentiert werden» (Terhart 1997, S. 36). Neben psychoanalytisch orientierten Verfahren fallen in diese Kategorie auch «solche rekonstruktiven Verfahren, die ohne ein auf die Handelnden gerichtetes Aufklärungsinteresse solche Strukturen zu dechiffrieren versuchen, die sich unabhängig vom Wissen und Wollen der Handelnden als universale generative Muster durchsetzen. In diesem Fall wird die Rekonstruktion der latenten Sinnstruktur völlig unabhängig von der subjektiven Repräsentanz vollzogen» (ebd.).

Ein solches forschungsmethodisches Vorgehen, das auf eine möglichst gegenstandsnahe Erfassung der ganzheitlichen Eigenschaften («qualia») des sozialen Feldes abzielt (vgl. ebd., S. 27), in Zusammenhang mit der Rolle von (digitalen) Medien in Lehr- und Lernprozessen zu bringen, stellt ein interessantes und viel versprechendes empirisches Unterfangen dar. Es bieten sich hier zahlreiche Möglich- 
keiten an, z. B. latente Strukturen gemeinsamer Lernprozesse oder geschlechtsspezifische Regeln beim gemeinsamen Arbeiten mit Computer und Internet zu rekonstruieren. Rekonstruktionslogische Untersuchungsvorhaben würden z.B. nicht die kognitiven bzw. individuellen Lernprozesse der Lernenden oder eine experimentelle Vergleichsuntersuchung der Wirksamkeit von neuen Medien auf den jeweiligen Lernerfolg ins Zentrum stellen.

Eine rekonstruktionslogische Perspektive auf Mediendidaktik einzunehmen heisst hier, die gemeinschaftlichen Lernprozesse von Lernenden in einer computerunterstützten Lernumgebung aus einer mikrosoziologischen Perspektive zu untersuchen bzw. untersuchen zu können.

Auf Grund des schulischen Kontexts lässt sich die vorliegende Untersuchung in den Bereich der qualitativen, rekonstruktiven oder interpretativen Schul- und Unterrichtsforschung verorten, die vor allem mit sozialwissenschaftlichen Methoden arbeitet (vgl. u. a. Breidenstein 2002). Diese Forschungsrichtung wurde im deutschsprachigen Raum in den 1970er Jahren von Terhart in Abgrenzung zu der bis dahin vorherrschenden standardisierten Unterrichtsbeobachtung programmatisch geprägt und zuletzt umfassender von Krummheuer/Naujok (1999) im Rahmen eines forschungsmethodischen Überblicks thematisiert. Allerdings liege noch kein einheitliches Konzept bzw. Forschungsgebiet einer qualitativ orientierten Schul- und Unterrichtsforschung vor (vgl. Breidenstein 2002, S. 24). Blickt man aber über die in diesem Rahmen vorhandenen unterschiedlichen Forschungsinteressen und theoretischen Perspektiven hinweg, lassen sich dennoch allgemeine Charakteristika feststellen. So hält Breidenstein als Gemeinsamkeit eine «sehr spezifische soziale Situation» fest, die allgemein als Unterricht gekennzeichnet werden kann. Dabei sei vor allem das «situierte interaktive Geschehen, das von den Teilnehmern als 〈Unterricht〉 definiert wird und zwar in seinem alltäglichen Vollzug und hinsichtlich seiner immanenten Strukturiertheit» (ebd., S. 12) von Interesse. Auf Grund der diffusen konzeptionellen Grundlage dieser Forschungsperspektive müsse sich (noch) jede konkrete Unterrichtsforschung sowohl theoretisch als auch methodisch neu verorten (vgl. ebd., S. 24). Eine solche Verortung wurde auch im Rahmen der empirischen Untersuchung mit der gewählten Methodologie und Methode vorgenommen.

So stellt die objektive oder strukturale Hermeneutik eine rekonstruktionslogische Methodologie dar, die die Chance (nicht nur) für mediendidaktische Fragestellungen bietet, vom Material ausgehend «Neues» zu entdecken als auch die Erfassung latenter Sinnstrukturen bzw. objektiver Handlungsstrukturen in Lernprozessen zu ermöglichen. Unter Verwendung dieser methodologischen und methodischen Perspektive kann eine qualitativ ausgerichtete empirische Untersuchung mit mediendidaktischem Fokus und mikrosoziologischer Perspektive auf alltäglichen Unterricht als rekonstruktionslogischer Beitrag einer qualitativ orientierten Schul- und Unterrichtsforschung im weitesten Sinne betrachtet werden. Daher werden die 
Grundannahmen der objektiven Hermeneutik im folgenden Abschnitt kurz umrissen, bevor das konkrete methodische Vorgehen der empirischen Untersuchung dargestellt wird.

\section{Zur Methodologie und Methode objektiven Hermeneutik}

Bei der objektiven oder auch strukturalen Hermeneutik, die auf Ulrich Oevermann et al. (vgl. 1979) zurückgeht, handelt es sich sowohl um eine Methodologie als auch eine Methode. Sie schlägt sich heute vor allem im Auswertungsverfahren der Sequenzanalyse nieder, die den rekonstruktionslogischen Anspruch dieser Methodologie forschungspraktisch repräsentiert. ${ }^{1}$ Im Folgenden werden für den vorliegenden Beitrag zentrale Aspekte zusammengefasst. ${ }^{2}$

Mit der Bezeichnung "objektive Hermeneutik» ist ein Konzept bzw. hermeneutisches Verfahren gemeint, das auf die Rekonstruktion objektiver Bedeutungs- bzw. latenter Sinnstrukturen von Handlungen zielt. Anliegen der objektiven Hermeneutik ist es, "auf wenig erforschten Gebieten und bei neuen, noch wenig bekannten Entwicklungen und Phänomenen, die typischen, charakteristischen Strukturen dieser Erscheinungen zu entschlüsseln und die hinter den Erscheinungen operierenden Gesetzesmässigkeiten ans Licht zu bringen» (Oevermann 2002, S. 1).

Im Wesentlichen wird mit dem Attribut «objektiv» betont, dass die subjektiven Intentionen der Textproduzenten nicht Gegenstand der jeweiligen Analyse sind bzw. sein können, sondern es immer (nur) möglich ist, die latenten Sinn- und objektiven Bedeutungsstrukturen ${ }^{3}$ des jeweiligen Gegenstandes zu rekonstruieren. Sinnstrukturiertheit wird von der objektiven Hermeneutik entsprechend als regelerzeugter

1 Oevermann et al. (vgl. 2002) plädieren dafür, statt zwischen qualitativer und quantitativer Forschung, zwischen Methoden auf der Ebene der Datenerhebung und -auswertung zu unterscheiden, so dass beide Prozeduren je getrennt nachvollzogen werden können. Demnach wird bei der Datenerhebung zwischen standardisierten und nicht-standardisierten Verfahren unterschieden, da der Aspekt der Authentizität und nicht der der Repräsentativität des Datenmaterials überwiegt, um möglichst gute Protokolle der Wirklichkeit für die Datenauswertung zu erhalten. So stellen z. B. technische Aufzeichnungen «natürliche» Protokolle dar, die noch nicht vorab durch Interpretationen im Sinne geprägt sind. Eine entsprechende terminologische Spezifizierung nimmt Oevermann auch auf der Ebene der Datenauswertung vor: Hier differenziert er zwischen subsumtionslogischen und rekonstruktionslogischen Verfahren. Oevermann (2002, S. 20) stellt in diesem Zusammenhang heraus, dass die Qualität der Forschung grundlegend von der Methode der Datenauswertung abhängt, weshalb im Rahmen der objektiven Hermeneutik als besonders wichtig erachtet wird, «die Probleme der Datenerhebung von den Problemen der Datenauswertung klar zu trennen», die in der üblichen Unterscheidung zwischen quantitativer und qualitativer Sozialforschung nicht deutlich werden.

2 Vgl. für eine ausführliche Darstellung und Diskussion der objektiven Hermeneutik u. a. Oevermann et al. 1979; Aufenanger/Lenssen 1986; Garz/Kraimer 1994; Kraimer 2000, Wagner 2001; Reichertz 2002; Oevermann 2002.

3 Bei latenten Sinn- und objektiven Bedeutungsstrukturen handelt es sich um «jene abstrakten, d. h. selbst sinnlich nicht wahrnehmbaren Konfigurationen und Zusammenhänge, die wir alle mehr oder weniger gut und genau 〈verstehen` und «lesen», wenn wir uns verständigen, Texte lesen, Bilder und Handlungsabläufe sehen, Ton- und Klangsequenzen hören und alle denkbaren Begleitumstände menschlicher Praxis wahrnehmen, die in ihrem objektiven Sinn durch bedeutungsgenerierende Regeln erzeugt werden und unabhängig von unserer je subjektiven Interpretation objektiv gelten» (Oevermann 2002, S. 2). 
objektiver Sinn gefasst, der die jeweiligen praktischen Handlungen und deren Objektivationen kennzeichnet.

Insofern ist ein unmittelbarer Zugriff auf Intentionen bzw. intentionale Gehalte nicht möglich, sondern diese Spuren sind nur über ihre «methodisch greifbare Verkörperung in Ausdrucksgestalten» (ebd., S. 2) erfassbar. Ausdrucksgestalten repräsentieren die psychischen, sozialen und kulturellen Erscheinungen einer konkreten, historischen Lebenspraxis, z. B. in Form einer Performanz, eines Kunstwerks oder eines Dialogs. In diesem Sinne fällt die Gesamtheit an Datenmaterial der erfahrbaren Welt in die für eine objektiv hermeneutische Forschungspraxis zentrale Kategorie der Ausdrucksgestalt. Sie können jedoch nur Gegenstand methodischer Rekonstruktion sein, wenn das zum Ausdruck Gebrachte grundsätzlich vertextbar ist und dieser Text sich forschungspraktisch als Protokoll behandeln lässt. ${ }^{4}$ Die Rekonstruktion von objektiven Bedeutungsstrukturen, die durch die Ausdrucksgestalten repräsentiert werden, geschieht in der Regel mit Hilfe des Verfahrens der Sequenzanalyse.

Das Verfahren der Sequenzanalyse "geht von der elementaren Feststellung aus, dass alle Erscheinungsformen von humaner Praxis durch Sequenziertheit strukturiert und konstituiert sind», wobei unter Sequenziertheit verstanden wird, dass «jegliches Handeln, selbst wenn es als monologisches oder individuell isoliertes in Erscheinung tritt, qua Regelerzeugtheit soziales Handeln ist» (Oevermann 2001, S. 30). Sie findet sich entsprechend in den Protokollen der authentischen Lebenspraxis wieder. ${ }^{5}$ Oevermann selbst bezeichnet das methodische Vorgehen der Sequenzanalyse als eine Kunstlehre. Folglich gibt es auch nicht das Verfahren der objektiv-hermeneutischen Textinterpretation. ${ }^{6}$

Es lassen sich dennoch theoretische Merkmale und prinzipielle Analyseschritte festhalten, die im Folgenden dargestellt werden und die in dieser Form den methodischen Kern der Auswertung der angeführten Untersuchung darstellen. Voraussetzung für die Analyse ist immer das Vorhandensein eines authentischen Protokolls sozialer Wirklichkeit bzw. der Lebenspraxis. Die Analyse beginnt damit,

4 Im Rahmen der objektiven Hermeneutik gilt ein methodologisch erweiterter Textbegriff, wonach prinzipiell alle Ausdruckgestalten menschlicher Praxis wie Musik, Bilder, Architektur, Bewegungen, Handlungen etc. vertextbar sind. Diesen weiten Textbegriff begründet Oevermann (vgl. 1986, S. 46 f.) mit der grundsätzlichen Textförmigkeit der Wirklichkeit und dem besonderen Verhältnis von Sprache und Handlung.

5 Elementar für die soziale Sequentialitätsstruktur ist, dass «jede konkrete Praxis im menschlichen Leben eröffnet und beschlossen werden muss, damit verbindlich und strukturiert gehandelt werden kann» (Oevermann 2002, S. 6) - ein viel angeführtes Beispiel hierfür stellt das alltägliche Begrüssungsritual dar.

6 Reichertz (vgl. 2002, S. 128 f.) hat in den bisherigen Veröffentlichungen zur objektiven Hermeneutik insgesamt fünf typische Formen der Forschungspraxis identifiziert, die von einer extensiven Feinanalyse bis zur Veranschaulichung der Ergebnisse in Form einer Glosse reichen. Bis heute hat sich die Sequenzanalyse durchgesetzt, die Reichertz als die «anspruchsvollste Variante der Objektiven Hermeneutik [beurteilt], da sie sich sehr stark an den methodologischen Prämissen des Gesamtkonzepts orientiert» (ebd., S. 129). 
dass innerhalb des vorliegenden Protokolls eine erste Interaktionseinheit von der bzw. dem Forschenden bestimmt und extensiv analysiert wird. Die Interpretation eines jeden Interakts entspricht vom Prinzip her einem Dreischritt, den Oevermann wie folgt zusammenfasst:

Die Rekonstruktion der objektiven Bedeutungsstruktur einer konkreten Äusserung beginnen wir im Rahmen der Objektiven Hermeneutik damit, dass wir zunächst Geschichten über möglichst vielfältige, kontrastierende Situationen erzählen, die konsistent zu einer Äusserung passen, ihre Geltungsbedingungen pragmatisch erfüllen. Im nächsten Schritt werden diese erzählten Geschichten, die implizite gedankenexperimentelle Konstruktionen darstellen, explizit auf ihre gemeinsamen Struktureigenschaften hin verallgemeinert, die in ihnen zum Ausdruck kommen, und im dritten Schritt werden diese allgemeinen Struktureigenschaften mit den konkreten Kontextbedingungen verglichen, in denen die analysierte Äusserung gefallen ist. (Oevermann 1983, S. 236 f.)

Dem folgt eine streng sequentielle kumulative Analyse der Interakte, d. h., Interakt für Interakt wird kontextfrei bzw. unter Einbezug des inneren Kontexts ausführlich und ohne Zeitdruck interpretiert bzw. der jeweilige Fall rekonstruiert. Mit der konsequenten Befolgung dieses Vorgehens findet zugleich eine methodische Kontrolle des Interpretationsprozesses statt. ${ }^{7}$

Die unter Verwendung des Verfahrens der Sequenzanalyse rekonstruierte Fallstruktur der jeweils vorliegenden Lebenspraxis mündet dann in die Formulierung einer Fallstrukturgesetzlichkeit. Aus den jeweiligen Fallrekonstruktionen mit ihren Fallstrukturgesetzlichkeiten lässt sich eine Strukturgeneralisierung folgern. ${ }^{8}$

Die besondere Chance wird in der Orientierung an der objektiven Hermeneutik darin gesehen, potenziell «Neues» entdecken zu können (vgl. u. a. Oevermann 2002). Denn diese strukturanalytische Herangehensweise ermöglicht es, Bekanntes aus einer anderen Perspektive zu erschliessen oder neu zu explizieren bzw. zu spezifizieren. Vor diesem Hintergrund wurde im Rahmen einer Untersuchung das mediendidaktische Themenfeld "Computer in der Grundschule», das seit etwa 20 Jahren vor allem unter einer praxisorientierten Perspektive aus medienpädagogischer Sicht diskutiert wird, empirisch mit dem Fokus gemeinschaftliche Lernen im Rahmen einer computerunterstützen Lernumgebung in den Blick genommen.

7 Dieses sehr grundsätzliche Vorgehen in drei Schritten (Erzählen von Geschichten - Bildung von Lesarten - Kontrastierung der Lesarten mit dem tatsächlichen Kontext) findet sich in differenzierter und erweiterter Form an anderen Stellen wieder (vgl. u. a. Wernet 2000; Reichertz 2002; Aufenanger/ Lenssen 1986; Oevermann 1983, 2002).

8 Auf die exemplarische Darstellung eines aussagekräftigen Beispiels für eine Sequenzanalyse muss hier aus Platzgründen und zugunsten der Schwerpunktsetzung dieses Beitrags auf die vorrangig theoretische Auseinandersetzung mit der rekonstruktionslogischen Forschung im Kontext der Mediendidaktik verzichtet werden. Vier ausführliche Darstellungen von Fallanalysen sind zu finden in Mayrberger 2007, S. 219-371). 


\section{Durchführung der Untersuchung und Zusammenschau der Ergebnisse}

Die Erhebung des Datenmaterials erfolgte, indem Grundschüler/innen in ihrem Unterrichtsalltag beim gemeinschaftlichen Arbeiten am Computer im geöffneten Unterricht während der Freiarbeitsphase beobachtet wurden. Die Dokumentation der Lernhandlungen wurde in Form einer videogestützten (Unterrichts-)Beobachtung insgesamt in zwei Schulklassen für jeweils eine Woche vorgenommen. Die erhobenen Bild- und Audiodaten entstanden sowohl durch Aufzeichnung der gemeinsamen Interaktion während der Lernprozesse mit einer Digitalvideokamera als auch durch die parallele Aufzeichnung des Monitorgeschehens mit einem Videorekorder (sog. Screenrecording). Im Anschluss wurde das Beobachtungsmaterial digital aufgearbeitet, so dass sich ein Video der beobachteten Situationen in Form einer Bild-in-Bild-Ansicht von Interaktions- und Monitorgeschehen mit dazugehöriger Tonspur ergab. Von ausgewählten Situationen gemeinschaftlicher Lernprozesse am und mit dem Computer wurden Transkripte erstellt, die das zu analysierende Protokoll darstellten. Dieses Material stellt die Grundlage für die theoriegeleitete Fallbestimmung im Sinne der Fragestellung(en) dar. Das ausgewählte Material wurde dann mit Hilfe der Sequenzanalyse analysiert, d. h., es wurden, entsprechend dem im vorherigen Abschnitt beschriebenen methodischen Vorgehen, die objektiven Bedeutungs- bzw. latenten Sinnstrukturen der gemeinschaftlichen Interaktionen der Schüler/innen beim Arbeiten mit dem Computer rekonstruiert.

Das Verfahren der Sequenzanalyse wurde für die Untersuchung begründet angepasst. ${ }^{9}$ Insgesamt wurden vier umfassende Fallanalysen vorgenommen. Im Zuge dieser liessen sich konstituierende Strukturelemente im Sinne einer Fallstrukturgeneralisierung für das gemeinschaftliche Lernen im Rahmen einer Medienecke im alltäglichen, geöffneten Unterricht der Grundschule rekonstruieren, die im Folgenden kurz dargestellt werden (vgl. ausführlicher Mayrberger 2007, S. 368 ff.):

- Die klare Regelung der Zusammenarbeit ist grundlegend für eine gemeinschaftliche Interaktion am Computer.

- Die Erfüllung von (extern gestellten) Arbeitsaufträgen stellt den Anlass und das Ziel von gemeinschaftlichen Interaktionen dar.

- Gemeinschaftliches Lernen (mit den neuen Medien) ermöglicht eine erfolgreichere Aufgabenbearbeitung für einzelne Teilnehmende am Interaktionsprozess als Einzelarbeit.

9 Dieses trifft in erster Linie die Notation, die auch Bildschirmfotos des Monitorgeschehens umfasst, sowie die Formulierung einer umfassenden Lesart. Es wurde im Vorfeld der Analyse die folgende übergreifende und theoriegestützte Lesart formuliert, die für alle Fälle gleichermassen gilt: Im Rahmen einer gemeinschaftlichen Interaktion in einer computerunterstützten Lernumgebung besteht auf Grund der Anlage der Lernumgebung für die Schüler/innen die Chance, den gemeinsamen Lernprozess im Sinne einer Ko-Konstruktion auszugestalten (vgl. Mayrberger 2007, S.208 ff.). 
- Gemeinschaftliche Interaktionsprozesse (am Computer) sind geprägt von der Koordination der gemeinsamen Handlungen auf inhaltlicher und organisatorischer Ebene.

- Im Zuge des formal gleichberechtigten gemeinschaftlichen Handelns lassen die Schüler/innen situationsbezogen Ungleichheit entstehen.

- Die gemeinschaftliche Kommunikation beim Arbeiten am Computer hat im Schwerpunkt die Aufgabenbearbeitung und/oder das Interaktionsverhältnis zum Gegenstand.

- Gemeinschaftliche Interaktionsprozesse (am Computer) zeichnen sich durch eine u. U. wechselnde Rollenaufteilung in Leit- und Co-Handelnde aus.

- Gemeinschaftliche Interaktionsprozesse zeichnen sich durch (temporäre) Phasen von Symmetrie und Asymmetrie im Interaktionsverhältnis aus.

- Beim gemeinschaftlichen Lernen mit den neuen Medien handelt es sich (vorwiegend) um kollaborative Lernprozesse.

- Computer und Internet beeinflussen den gemeinschaftlichen Lernprozess durch die (gemeinsame) Zuschreibung bzw. Akzeptanz als Macht- oder Lernmittel massgeblich.

Aus den konstituierenden Strukturbedingungen gemeinschaftlicher Lernprozesse lassen sich drei zentrale Dimensionen erschliessen, die für ein gemeinschaftliches Lernen in Medienecken im alltäglichen, geöffneten Unterricht der Grundschule konstitutiv sind:

(1) Dimension Interaktionsverhältnis: Symmetrie - Asymmetrie

(2) Dimension Kommunikationsverhalten: Diskurs - Ignorieren

(3) Dimension Funktionen neuer Medien: Lernmittel - Machtmittel

Im Zuge der weiteren Analyse der Ergebnisse wurden die drei Dimensionen mit Hilfe eines Achsenkreuzes in Beziehung gesetzt und die vier Fälle dort exemplarisch eingeordnet. So lässt sich ein Bereich identifizieren, innerhalb dessen Prozesse «veränderten Lernens» im Sinne von Ko-Konstruktion wahrscheinlich sind: Ein «neues Lernen» im gemeinschaftlichen Lernprozess ermöglichen potenziell ein Zusammenwirken von starker Symmetrie in der Interaktion und eine diskursorientierte Auseinandersetzung mit der Problemstellung während der gemeinsamen Arbeit am Computer sowie die Verwendung der neuen Medien als Lernmittel oder -werkzeug.

Knapp zusammengefasst lässt sich mit Bezug auf die leitenden Fragestellung(en) aus den empirischen Ergebnissen folgern, dass im Rahmen eines alltäglichen, geöffneten Unterrichts der Grundschule unter Verwendung von Medienecken ein «verändertes Lernen» im Sinne von Ko-Konstruktion (noch) nicht in vollem Umfang stattfindet (vgl. ausführlicher Mayrberger 2007, S. 373 ff.) 
Nachdem in groben Zügen der Gang der Untersuchung und ein Überblick der wesentlichen Erkenntnisse dargestellt wurde, werden im Folgenden vor diesem Hintergrund die Stärken und Schwächen einer rekonstruktionslogischen Forschung in Form der objektiven Hermeneutik im mediendidaktischen Kontext erörtert.

\section{Potenzial und Grenzen einer rekonstruktionslogischen Forschungs- perspektive am Beispiel der objektiven Hermeneutik für die Mediendidaktik}

Die objektive Hermeneutik betont ihr Potenzial für die jeweilige Praxis, der der jeweilige Fall entstammt (vgl. u. a. Oevermann 2002), d. h. im vorliegenden Fall für das gemeinschaftliche Lernen in Medienecken im geöffneten Grundschulunterricht. Für die Pädagogik sieht Kraimer (vgl. 2000) die besonderen Chancen in der Anwendung der objektiven Hermeneutik darin, dass die im Rahmen einer Fallrekonstruktion gewonnenen Einsichten sowohl zu einer gegenstandsspezifischen Theoriebildung beitragen als auch die berufliche Praxis verändern können. In diesem Sinne stellen die mit Hilfe der Sequenzanalyse rekonstruierten Ergebnisse sowohl einen theoretischen Beitrag zur grundlagen- als auch (begrenzt) zur praxisorientierten Forschung im Bereich der Mediendidaktik bzw. der Integration von neuen Medien im Unterricht der Grundschule dar.

Im Rahmen des empirischen Forschungsprojekts wurde sowohl bei der Auseinandersetzung mit der Methodologie als auch bei der Anwendung der Sequenzanalyse festgestellt, dass, insgesamt betrachtet, die objektive Hermeneutik für den vorliegenden Gegenstand eine angemessene Zugangsweise darstellt. Dieses wird im Folgenden anhand einiger Erkenntnisse aus dem Forschungsprozess kritisch erörtert.

Im Verlauf der Analyse hat sich praktisch gezeigt, dass das digitale Screenrecording ein standardisiertes Verfahren zu Datenerhebung darstellt, das es ermöglicht, eine komplexe Analyse computerunterstützter Interaktion vorzunehmen. Es konnten auf diese Weise geeignete Protokolle für die Auswertung mittels Sequenzanalyse erstellt werden, die dem methodologischen Anspruch der Authentizität gerecht werden.

Die Stärke der objektiven Hermeneutik liegt für die gewählte Fragestellung in ihrer strukturalistischen Ausrichtung. Im Gegensatz zu anderen qualitativen Zugangweisen im Kontext von Grundschule, die z. B. vornehmlich den subjektiv gemeinten Sinn der Lernenden herausarbeiten bzw. interpretativ nachvollziehen wollen und damit das fokussieren, was die Lernenden beabsichtigten auszudrücken, ermöglicht es die Sequenzanalyse aus den Protokollen der Interaktion zur rekonstruieren, was die Lernenden im Zuge ihrer Lernprozesse ausgedrückt haben. So hat sich in der konkreten Forschungspraxis gezeigt, dass die Sequenzanalyse dafür geeignet ist, Lernprozesse aus einer qualitativen Perspektive in den Blick zu nehmen, d. h. für den vorliegenden Fall, gemeinschaftliche Lernprozesse im Sinne beobachtbarer Lernhandlungen im Rahmen von Interaktion von Schülern/-innen aus einer 
mikroanalytischen Perspektive zu rekonstruieren und brauchbare Aussagen zu generieren.

Ergänzend zur hier behandelten Fragestellung liessen sich mit der Sequenzanalyse im Rahmen von mediendidaktischen Fragestellungen z. B. auch die objektiven Bedeutungsstrukturen von computerunterstützten Lernpfaden oder Bildungsprozessen von Lernenden rekonstruieren. Dazu würde als weitere Notationsvariante die Logdatei, die Prozesse bzw. Aktionen der bzw. des Lernenden am Computer protokolliert, zur Analyse herangezogen. Auf dieses Weise könnten im Gegensatz zum vorliegenden Untersuchungsdesign auch individuelle Lernprozesse unabhängig von einer verbalen Interaktion rekonstruiert werden. Zudem bieten die im Rahmen der Untersuchung ausführlich durchgeführten Fallrekonstruktionen mit ihren Ergebnissen einen relativ detaillierten Einblick in gemeinschaftliche, computerunterstützte Lernprozesse, an die in weiteren Untersuchungen angeknüpft werden kann.

Dass die Sequenzanalyse auch ihre Grenzen hat, wird im Folgenden deutlich: Es konnten mit Hilfe dieser im Rahmen der Fallrekonstruktionen zwar Strukturen gemeinschaftlichen Lernens herausgearbeitet werden, aus denen die Beantwortung der Frage nach dem Inwiefern eines «neuen Lernens» mit «neuen Medien» gefolgert werden konnte sowie Bedingungen rekonstruiert werden, die für einen gemeinschaftlichen Lernprozess im Sinne von Ko-Konstruktion förderlich sind, doch um diese Ergebnisse für die konkrete mediendidaktische Praxis fruchtbar zu machen, bedarf es Folgeuntersuchungen, um über praktische Folgerungen auf einer sehr allgemeinen Ebene hinausgehen zu können. Im Zuge solcher Untersuchungen sollte überlegt werden, inwiefern dem Anspruch einer Erforschung von alltäglichem Unterricht weiterhin nachgekommen werden kann oder ob es z. B. aus forschungsökonomischen Gründen und methodischen Überlegungen heraus sinnvoller wäre, Einfluss auf den Untersuchungsgegenstand, in diesem Fall dem alltäglichen Unterricht unter Einbezug von Medienecken, zu nehmen. So könnten z.B. bewusst Situationen durch eine gezielte Veränderung der Rahmenbedingungen erzeugt werden, in denen über den Gegenstand und das gemeinsame Vorgehen im Sinne der rekonstruierten förderlichen Situation für ein verändertes Lernen kommuniziert werden muss und die Interaktion möglichst symmetrisch verläuft. Eine solche Veränderung kann bei der Aufgabenstellung und Softwareauswahl (z. B. problemorientierte und situierte Aufgaben), einer gezielte Gruppenzusammensetzung (z. B. mit Unterstützung eines zuvor erstellen Soziogramms der Klasse oder nach leistungsbezogenen Kriterien) oder in Form einer optimalen, technischen Ausstattung (z.B. schneller Internetzugang, zwei Computermäuse) erfolgen. Auch bietet es sich im Kontext von Anschlussuntersuchung an, den explizit allgemein mediendidaktischen Fokus auf Grundlage von fachdidaktischen Erkenntnissen und Erfordernissen zu erweitern bzw. zu spezifizieren. Da eine Untersuchungsanlage dieser Ausrichtung in Teilen experimentell orientiert wäre, be- 
dürfte es auf Grund der veränderten methodischen Rahmenbedingungen einer Anpassung der Fragestellung. Inwieweit ein rekonstruktionslogisches Vorgehen bei der Auswertung der erhobenen Daten methodologisch begründbar und zielführend wäre, bliebe zu überprüfen.

Zusammenfassend lässt sich feststellen, dass eine rekonstruktionslogische Herangehensweise im Sinne der objektiven Hermeneutik es ermöglicht, einen schon (relativ) bekannten Gegenstand, hier die Integration der neuen Medien in den Unterricht der Grundschule, methodisch kontrolliert (wieder) neu in den Blick zu nehmen. Sie bietet die Chance, Lehr- und Lernprozesse ganzheitlicher zu betrachten, als es z.B. im Rahmen standardisierter Herangehensweisen geschieht, die z.B. die spezifischen Veränderungen des individuellen Leistungsstandes zu einem bestimmten Zeitpunkt messen. Insofern birgt eine rekonstruktionslogische Perspektive auf Lernprozesse, wenn sie als soziale Interaktion verstanden werden, das Potenzial, zur Erweiterung des Erkenntnisspektrums innerhalb der Lehr-Lernforschung und empirischen Bildungsforschung beizutragen.

\section{Fazit}

Im Rahmen des vorliegenden Beitrags wurde aufgezeigt, dass eine rekonstruktionslogische Perspektive auf das Lehren und Lernen mit neuen Medien (im Rahmen von Medienecken im Grundschulunterricht) einen wichtigen und zielführenden empirischen Zugang darstellt, der begründet als Alternative und Ergänzung zu hypothesenprüfenden und standardisierten Verfahren im Bereich der medienbezogenen Lehr- und Lernforschung betrachtet werden kann.

Der rekonstruktionslogische Zugang, der sich methodisch vor allem in der Verwendung der objektiven Hermeneutik in Form der Sequenzanalyse äussert, ermöglicht es, neue empirische Erkenntnisse zu einem schon bekannten Feld zu gewinnen. Die Ergebnisse der Untersuchung stellen einen empirischen Beitrag zur (weiteren) Auseinandersetzung mit dem Themenfeld «Neue Medien in der Grundschule» aus einer mediendidaktischen Perspektive dar und bieten interessante Anschlussmöglichkeiten für weitere empirische Untersuchungen in diesem Themenfeld.

\section{Literatur}

Aufenanger, Stefan. «Lernen mit neuen Medien - Was bringt es wirklich? Forschungsergebnisse und Lernphilosophien.» Medien praktisch, Jg. 23, H. 92 (1999): 4-8.

Aufenanger, Stefan; Lenssen, Margrit. «Einleitung: Zum Problem der objektiven Hermeneutik.» Handlung und Sinnstruktur: Bedeutung und Anwendung der objektiven Hermeneutik. Hrsg. v. Stefan Aufenanger und Margrit Lenssen. München: Kindt, 1986. 1-18.

Bohnsack, Ralf. Rekonstruktive Sozialforschung: Einführung in qualitative Methoden. 5. Aufl. Opladen: Leske + Budrich, 2003.

Breidenstein, Georg. «Interpretative Unterrichtsforschung - eine Zwischenbilanz und einige Zwischenfragen.» Forum qualitative Schulforschung 2: Interpretative Unterrichts- und 
Schulbegleitforschung. Hrsg. v. Georg Breidenstein. Opladen: Leske + Budrich, 2002. $11-28$.

Dörr, Günter; Strittmatter, Peter. «Multimedia aus pädagogischer Sicht.» Information und Lernen mit Multimedia und Internet: Lehrbuch für Studium und Praxis. 3., vollst. überarb. Aufl. Hrsg. v. Ludwig Issing und Paul Klimsa. Weinheim: Beltz PVU, 2002. 29-42.

Flick, Uwe. Qualitative Forschung: Theorie, Methoden, Anwendung in Psychologie und Sozialwissenschaften. 4. Aufl. Reinbek bei Hamburg: Rowohlt, 1999.

Flick, Uwe; v. Kardorff, Ernst; Steinke, Ines. «Was ist qualitative Forschung? Einleitung und Überblick.» Qualitative Forschung: Ein Handbuch. Hrsg. v. Uwe Flick, Ernst v. Kardoff und Ines Steinke. Reinbek bei Hamburg: Rowolth, 2000. 13-29.

Garz, Detlef; Kraimer, Klaus (Hrsg.). Die Welt als Text: Theorie, Kritik und Praxis der Objektiven Hermeneutik. Frankfurt a. M.: Suhrkamp, 1994.

Hüther, Jürgen. «Mediendidaktik.» Grundbegriffe Medienpädagogik. 4., vollst. neu konzipierte Aufl. Hrsg. v. Jürgen Hüther und Bernd Schorb. München: kopaed, 2005. 234240.

Hüther, Jürgen; Schorb, Bernd (Hrsg.). Grundbegriffe Medienpädagogik. München: kopaed, 2005.

Kraimer, Klaus (Hrsg.) Die Fallrekonstruktion: Sinnverstehen in der sozialwissenschaftlichen Forschung. Frankfurt a. M.: Suhrkamp, 2000.

Kron, Friedrich W.; Sofos, Alivisos. Mediendidaktik: Neue Medien in Lehr- und Lernprozessen. München: Reinhardt, 2003.

Krummheuer, Götz; Naujok, Natalie. Grundlagen und Beispiele Interpretativer Unterrichtsforschung. Opladen: Leske + Budrich, 1999.

Mayrberger, Kerstin. Verändertes Lernen mit neuen Medien? - Strukturanalyse gemeinschaftlicher Interaktionen in einer computerunterstützten Lernumgebung in der Grundschule. Hamburg: Kovac, 2007.

Mitzlaff, Hartmut (Hrsg.). Internationales Handbuch Computer (ICT), Grundschule, Kindergarten und Neue Lernkultur. 2 Bände. Baltmannsweiler: Schneider-Verlag Hohengehren, 2007.

Oevermann, Ulrich. «Zur Sache. Die Bedeutung von Adornos methodologischem Selbstverständnis für die Begründung einer materialen soziologischen Strukturanalyse.» AdornoKonferenz: 1983. Hrsg. v. Ludwig von Friedeburg und Jürgen Habermas. Frankfurt a. M.: Suhrkamp, 1983. 234-289.

Oevermann, Ulrich. «Kontroversen über sinnverstehende Soziologie. Einige wiederkehrende Probleme und Missverständnisse in der Rezeption der «Objektiven Hermeneutik» Handlung und Sinnstruktur: Bedeutung und Anwendung der Objektiven Hermeneutik. Hrsg. v. Stefan Aufenanger und Margrit Lenssen. München: Kindt, 1986. 19-83.

Oevermann, Ulrich. Strukturprobleme supervisorischer Praxis: Eine objektiv hermeneutische Sequenzanalyse zur Überprüfung der Professionalisierungstheorie. Frankfurt a. M.: Humanities Online, 2001.

Oevermann, Ulrich. «Klinische Soziologie auf der Basis der Objektiven Hermeneutik - Manifest der objektiv hermeneutischen Sozialforschung.» März 2002. Internet: «http://www.agoh.de/cms/index.php?option=com_remository\&ltemid=293\&func=fileinf o\&id=28> (5.11.2007).

Oevermann, Ulrich; Allert, Tilmann; Konau, Elisabeth; Krambeck, Jürgen. «Die Methodologie einer ,objektiven Hermeneutik' und ihre allgemeine forschungslogische Bedeutung in den Sozialwissenschaften.» Interpretative Verfahren in den Sozial- und Textwissenschaften. Hrsg. v. Hans-Georg Soeffner. Stuttgart: Metzler, 1979. 352-434. 
Reichertz, Jo. «Die objektive Hermeneutik - Darstellung und Kritik.» Qualitative Forschung: Grundlagen und Methoden. 2., vollst. überarb. Aufl. Hrsg. v. Eckard König und Peter Zedler. Weinheim: Beltz, 2002. 123-156.

Reinmann-Rothmeier, Gabi; Mandl, Heinz. «Unterrichten und Lernumgebungen gestalten.»Pädagogische Psychologie: Ein Lehrbuch. 4., vollst. überarb. Aufl. Hrsg. v. Andreas Krapp und Bernd Weidenmann. Weinheim: Beltz PVU, 2001. 601-646.

Reusser, Kurt. "Co-constructivism in Educational Theory and Practice.» International Encyclopedia of the Social and Behavioral Sciences. Hrsg. v. Neil J. Smelser, Paul B. Baltes und Franz E. Weinert. Oxford: Pergamon/Elsevier Science, 2001. 2058-2062.

Sacher, Werner. Schulische Medienarbeit im Computerzeitalter: Grundlagen, Konzepte und Perspektiven. Bad Heilbrunn/Obb.: Klinkhardt, 2000.

Schulz-Zander, Renate. «Veränderung der Lernkultur mit digitalen Medien im Unterricht.» Perspektiven der Medienpädagogik in Wissenschaft und Bildungspraxis. Hrsg. v. Hubert Kleber. München: kopaed, 2005. 125-140.

Schwetz, Herbert; Zeyringer, Manuela; Reiter, Anton (Hrsg.). Konstruktives Lernen mit neuen Medien: Beiträge zu einer konstruktivistischen Mediendidaktik. Innsbruck: Studien-Verlag, 2001.

Terhart, Ewald. «Entwicklung und Situation des qualitativen Forschungsansatzes in der Erziehungswissenschaft.» Handbuch Qualitative Forschungsmethoden in der Erziehungswissenschaft. Hrsg. v. Barbara Friebertshäuser und Annedore Prengel. Weinheim: Juventa, 1997. 27-42.

Tulodziecki, Gerhard; Herzig, Bardo. Mediendidaktik: Medien in Lehr-Lernprozessen. Stuttgart: Klett-Cotta, 2004.

Wagner, Hans-Josef. Objektive Hermeneutik und Bildung des Subjekts. Weilerswist: Velsbrück Wissenschaft, 2001.

Wernet, Andreas. Einführung in die Interpretationstechnik der Objektiven Hermeneutik. Opladen: Leske + Budrich, 2000. 